\title{
Fast reconstruction algorithm dealing with tomography artifacts
}

\author{
Federica Marone*a, Beat Münch ${ }^{\mathrm{b}}$, Marco Stampanoni ${ }^{\mathrm{a}, \mathrm{c}}$ \\ ${ }^{a}$ Swiss Light Source, Paul Scherrer Institut, 5232 Villigen, Switzerland; \\ ${ }^{\mathrm{b}}$ Empa Materials Science and Technology, 8600 Dübendorf, Switzerland; \\ ${ }^{c}$ Institute for Biomedical Engineering, University and ETH Zürich, 8092 Zurich, Switzerland
}

\begin{abstract}
Highly brilliant X-rays delivered by third generation synchrotron facilities coupled with modern detector technology permit routinely acquisition of high resolution tomograms in few minutes, making high throughput experiments a reality and bringing real-time tomography closer. New solutions for fast post-processing of such large amount of data are mandatory to fully exploit advantages provided by the high acquisition speed enabling new experiments until recently even unimaginable.

The TOMCAT beamline ${ }^{1}$ is well equipped for fast and high throughput experiments ${ }^{2,3}$. Here, we will focus on our solutions regarding the reconstruction process and discuss a fast reconstruction algorithm ${ }^{4}$, based on the Fourier Transform method as opposed to slower standard Filtered Back-Projection routines. We perform the critical step of such method, the polar-to-Cartesian mapping in the Fourier space, by convolution with the Fourier transform of functions with particular characteristics. This convolution approach combines speed with accuracy, making real-time data postprocessing closer to reality.

This fast reconstruction algorithm implemented at TOMCAT also features several plug-ins, aimed at taming reconstruction artifacts. Here, we will discuss a new approach for removing rings from reconstructed datasets arising from defective detector pixels and/or damaged scintillator screens. This new method is based on a combined waveletFFT decomposition ${ }^{5}$. Another important feature of the presented reconstruction algorithm deals with local tomographic datasets, characterized by incomplete data. We show here that ad-hoc padding of the sinograms prior to reconstruction significantly reduces typical artifacts related to data incompleteness, making local tomography a valuable acquisition mode when small volumes in relatively large samples are of interest.
\end{abstract}

Keywords: synchrotron radiation, tomographic microscopy, real-time, reconstruction algorithms, Fourier methods, ring artifacts, local tomography

\section{INTRODUCTION}

The TOMCAT (TOmographic Microscopy and Coherent rAdiology experimenTs) beamline ${ }^{1}$ at the Swiss Light Source (SLS), with its automated sample exchanger ${ }^{2}$, fast data acquisition protocol ${ }^{6}$ and semi-automated post-processing approach ${ }^{7}$, is well equipped for high throughput experiments. Typically, high resolution datasets can be acquired with the standard setup in 5-15 minutes depending on the used beam energy. Ongoing work focuses on the implementation of ultrafast tomography at the beamline with the goal of acquisition of full tomograms in $1 \mathrm{~Hz}$ tact $^{3}$. Post-processing and tomographic reconstruction of these raw datasets should ideally occur in a similar time scale as their acquisition, so that data collection and reconstruction can go on parallel, allowing quality checks and data evaluation shortly after the end of a scan. The post-processing pipeline currently implemented at TOMCAT $^{7}$ takes about 15 minutes for the reconstruction of a full 3D high resolution volume and therefore is well suited for the actual acquisition setup and speed. In particular, a selection of sinograms is computed on-the-fly during acquisition, so that immediately after a scan, single slices can be reconstructed and the required parameters (center of rotation, filter, ...) tuned using a web interface ${ }^{7}$. The full reconstruction job, from raw projections to reconstructed slices, is then run on a Linux cluster. To be able to handle and post process in a reasonable time frame the large amount of data, which are produced by $1 \mathrm{~Hz}$ tomographic scans, it is desirable to speed up this reconstruction process.

*federica.marone@psi.ch; phone +4156310-5318

Developments in X-Ray Tomography VII, edited by Stuart R. Stock, Proc. of SPIE Vol. 7804, $780410 \cdot$ @ 2010 SPIE · CCC code: $0277-786$ X/10/\$18 - doi: 10.1117/12.859703 
In this paper, we present our solutions regarding the reconstruction of single slices from sinograms and discuss a fast algorithm, based on the Fourier Transform method as opposed to slower standard Filter Back-Projection (FBP) routines. In addition, we also illustrate how this fast reconstruction algorithm deals with ring and local tomography artifacts, delivering high quality reconstructions.

\section{FOURIER METHODS}

According to the Fourier slice theorem ${ }^{8}$, the Fourier transform of a parallel projection of an object obtained at angle $\theta$ equals a line of the 2D Fourier transform of the object taken at the same angle. Making use of this theorem, the 2D Fourier space can be filled with the Fourier transforms of parallel projections of an object taken at different angles. Information on the linear absorption coefficient of the study object can then be recovered by a 2D inverse Fourier transform of the Fourier space, if this is sufficiently sampled. Hence, such tomographic reconstruction process consists in a series of 1D Fourier transforms followed by a 2D inverse Fourier transform.

The advantage of such an approach lies in the possibility of using the Fast Fourier Transform (FFT) to perform the inverse 2D Fourier transform in a number of steps on the order of $N^{2} \log N$ for an $N x N$ array, as opposed to $n_{\text {angle }} x N^{2}$ for standard FBP algorithms. Despite this advantage, Fourier Transform methods have been so far not widely exploited. The reason lies in the difficulty of interpolating information in the Fourier space from the polar to the Cartesian grid required for efficient computation of the 2D inverse FFT.

Most interpolation techniques (linear, bilinear, splines, ...) are not sufficiently accurate and with reasonable computational efforts the quality of FBP reconstructions is not achieved. As an alternative, the algorithm presented here, gridrec, initially introduced by Down et al. (1999) ${ }^{4}$, makes use of the gridding method, presently considered to be the most accurate Fourier reconstruction technique.

The gridding approach was originally proposed in radio astronomy to back-transform irregularly sampled Fourier data and later introduced in computerized tomography by O'Sullivan $(1985)^{9}$. In the gridding technique, the data in the Fourier space are mapped onto a Cartesian grid not by simple interpolation, but by convolution with the Fourier transform of a certain function $w(x, y)$, whose contribution is removed after the $2 \mathrm{D}$ inverse FFT. For best performance and reconstruction accuracy, this function $w(x, y)$ needs to be well concentrated in the region of interest and its Fourier transform should vanish for spatial frequencies larger than a few grid spacings. In the implementation presented here, a separable form for $w(x, y)=w(x) w(y)$ is used, with $w(x)$ chosen from the family of 1D Prolate Spheroidal Wave Functions (PSWF) of zeroth order ${ }^{10}$. These functions and their Fourier transforms can be efficiently computed and stored at run time, using known rapidly converging expansions of PSWF in terms of Legendre polynomials ${ }^{11}$.

\subsection{Artifacts and their suppression}

Computer implementations of tomographic reconstruction algorithms, based both on Fourier methods and FBP routines, can lead to several artifacts (interperiod interference and dc shift) in the reconstructed images ${ }^{8,12}$, if the nature of the circular convolution and the discretization of the truncated filter kernel are not properly taken into account. Although the recognition of these artifacts and the possibilities for their suppression are not new, in standard implementations of reconstruction algorithms (e.g. iradon function in Matlab $®$ ) these issues are nonetheless often neglected.

Interperiod interference is caused by the implementation of the aperiodic convolution needed by the filtering operation as a periodic convolution as required by the discretized problem with bandlimited projections and finite spatial support. This artifact can easily be overcome by adequately zero-padding the projections. The minimum number of added zeroes must equal the number of samples in the original projection minus 1.

A constant offset in the grey level values throughout a reconstructed slice is instead related to the discretization of the truncated filter kernel. In fact, the discrete Fourier transform of the finite and discrete impulse response of the filter kernel (e.g. band-limited ramp filter) is different from the continuous filter itself (Figure 1). The main difference lies in the dc component responsible for the observed constant offset.

\subsection{Accuracy assessment}

To assess the accuracy of the reconstructions obtained with gridrec, we chose the well known Shepp-Logan phantom ${ }^{13}$, introduced in 1974 and still in common use today. We generated with Matlab ${ }^{\circledR}$ a Modified Shepp-Logan phantom 
(Figure 2(a)) with 2048x2048 pixels, featuring improved contrast compared to the standard phantom, allowing better visual perception. Subsequently, we created the corresponding sinogram with 1501 different views.
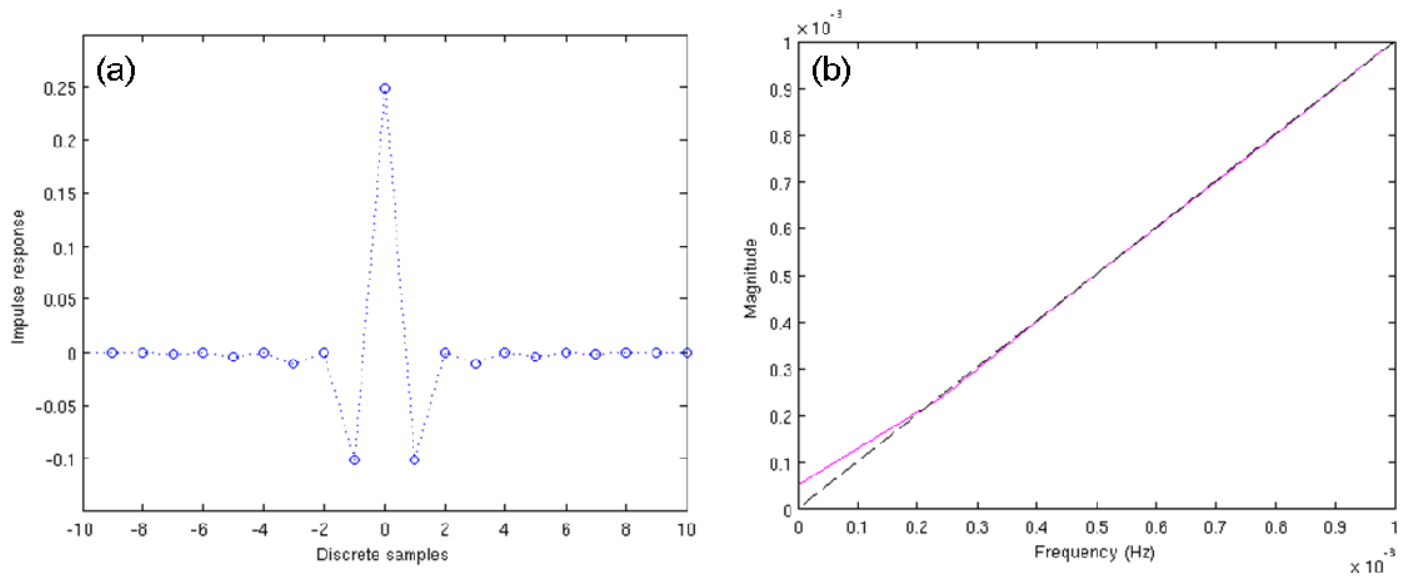

Figure 1: Filter kernel - (a) Finite and discrete impulse response of the bandlimited ramp filter (b) Comparison between $|\omega|$ (dashed black) and the Fourier transform of the function shown in (a) (pink) in the vicinity of the origin.

This sinogram has been reconstructed (Figure 2) with gridrec: (c) with no zero padding, (d) with moderate padding $(\mathrm{Z}=0.5$, width of the sinogram array extended left and right by half field of view and filled with zeroes) and (e) with major padding $(\mathrm{Z}=1.5)$. While gridrec reconstructions with zero padding (Figure 2(d)-(e)) are qualitatively comparable with the original phantom (Figure 2(a)), gridrec reconstructions without padding (Figure 2(c)) show significant aliasing artifacts (section 2.1) in particular at the margins of the image. As comparison, a reconstruction with standard $\mathrm{FBP}^{14}$ $(Z=1.5)$ is also shown (Figure 2(b)).
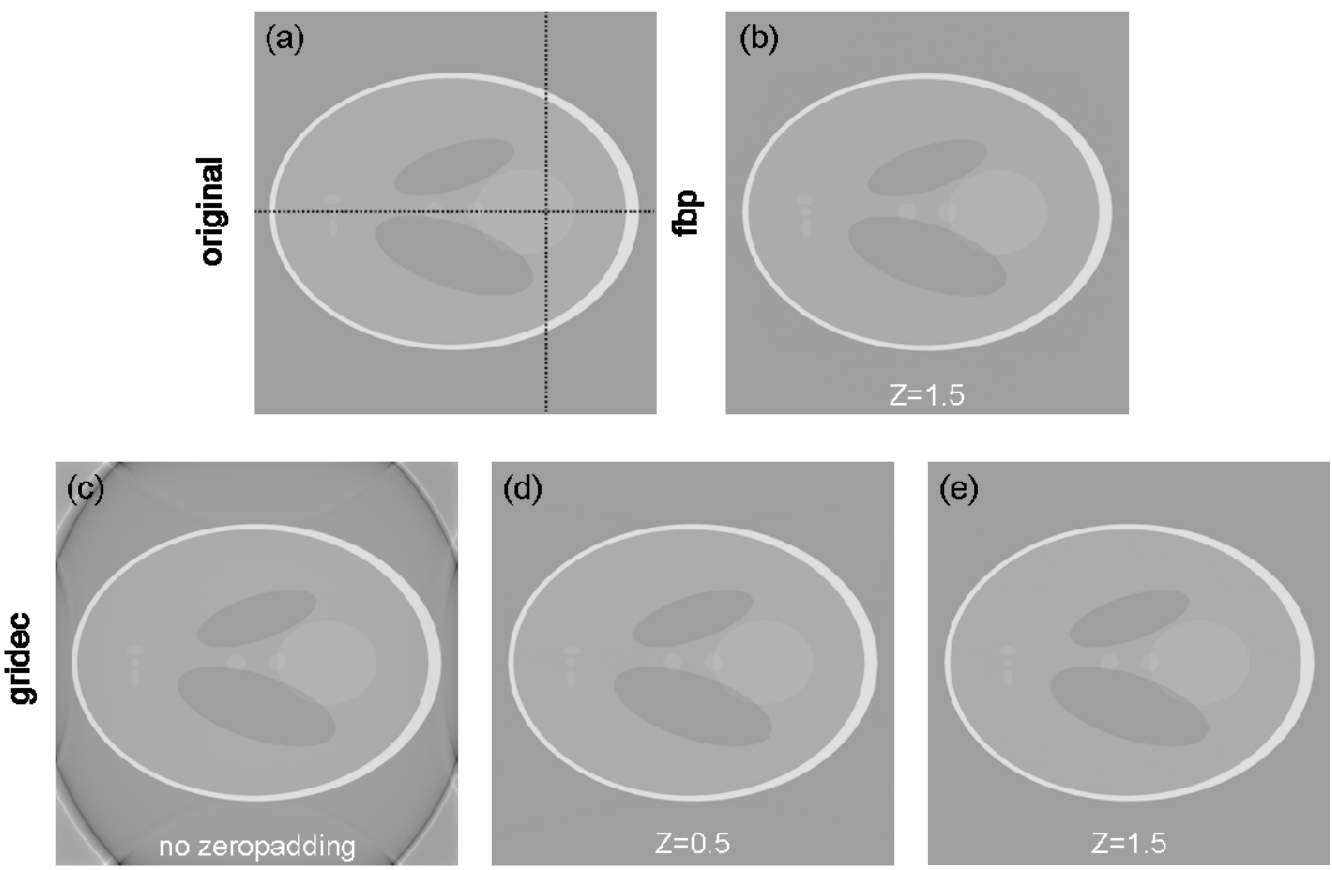

Figure 2: Modified Shepp-Logan phantom (a) and reconstructions with FBP (b) and gridrec with different zero padding modalities ((c)-(e)). The dotted lines in (a) indicate where the profiles in Figure 3 are taken. 
(a)

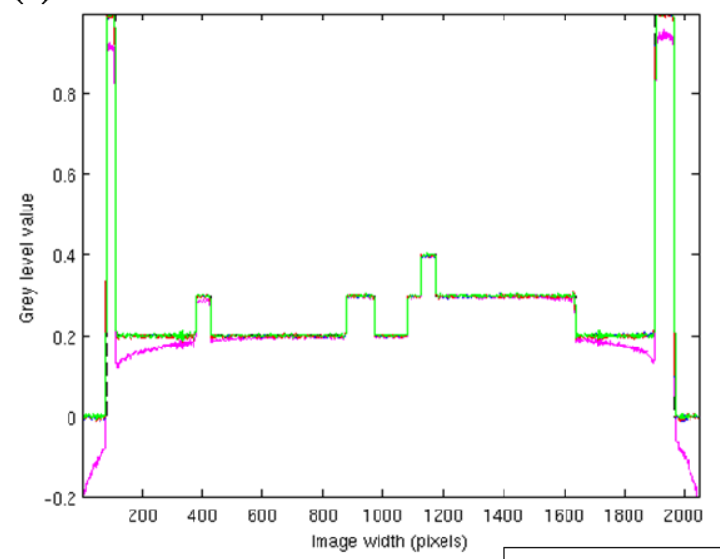

(b)

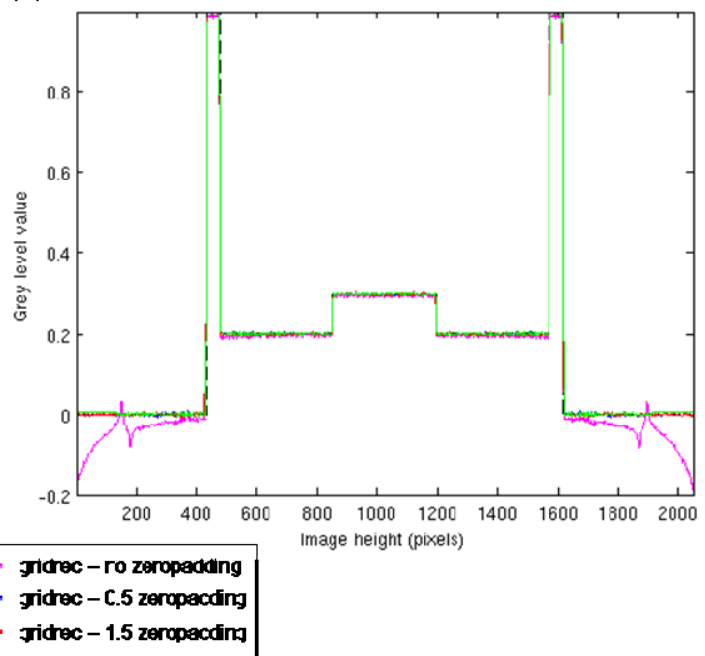

Figure 3: Line profiles through the original phantom (dashed black line) and the reconstructions obtained with FBP (green) and gridrec (pink, blue and red for increasing zero padding) along the horizontal (a) and vertical (b) dotted lines in Figure 2(a).

(a)
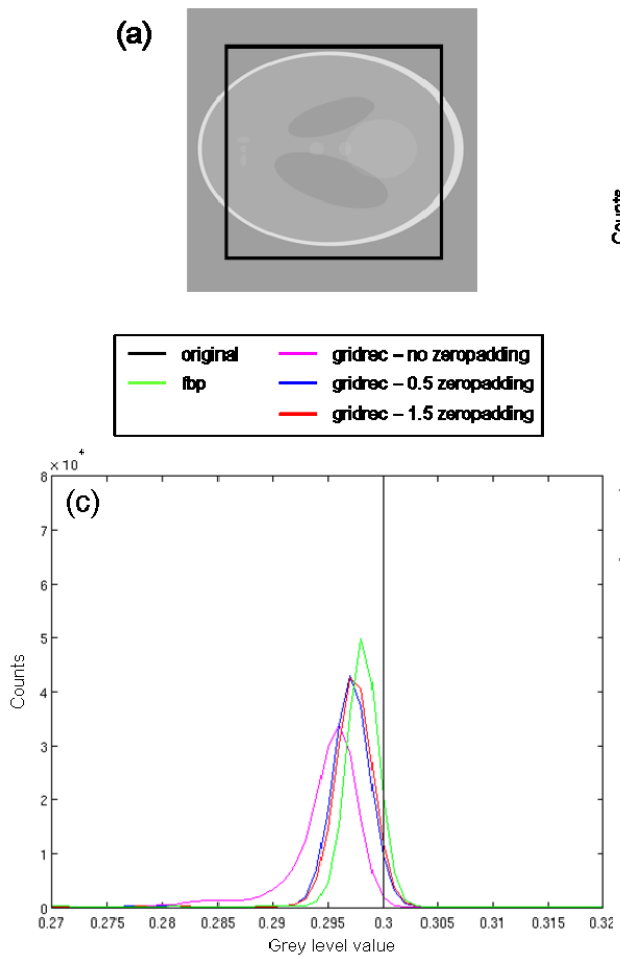
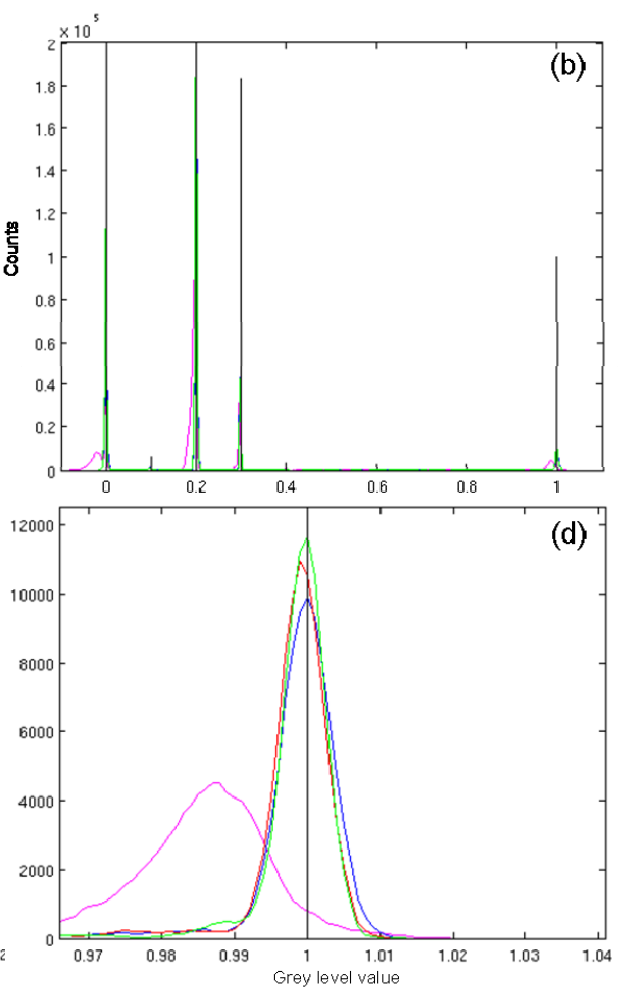

Figure 4: Grey level value histograms for the square region in (a) for the original phantom (black line) and the reconstructions with the different algorithms (colored lines) - (b) Overview, (c-d) Zoom in on two major peaks. 
For a more quantitative assessment of the accuracy of the reconstructions obtained with gridrec, in Figure 3 profiles along the dotted lines in Figure 2(a) are shown. The line for the original phantom, for the FBP and gridrec (with padding) reconstructions are all overlapping below the green line.

Reconstructions with gridrec without zero padding (pink line) show poor quality. In addition to the aliasing artifacts already clearly visible in Figure 2(c), a cupping artifact is also observed in the profile plots (Figure 3), making any quantitative analysis of the data difficult. The severity of these artifacts depends on the amount of field of view occupied by the study object and is smaller for objects occupying only minor portions of the image area, since such a situation would be comparable to a reconstruction with a certain amount of zero padding. These artifacts, however, completely disappear when zero padding is used. As stated by the theory (section 2.1), moderate zero padding $(\mathrm{Z}=0.5)$ is sufficient to eliminate interperiod interference.

Figure 3 clearly shows that the results obtained with FBP (green) and gridrec with zero padding (blue and red) are almost equivalent, demonstrating that Fourier methods can provide high quality reconstructed datasets with an accuracy comparable to that guaranteed by standard FBP routines. Since in the used FBP algorithm ${ }^{14}$, the filter kernel is not properly implemented $(|\omega|$ is used instead of the pink curve in Figure 1(b)), to be able to compare results, in Figure 3 an artificial constant offset $(0.0042)$ has been added to the (green) line for the FBP reconstructions.

The detailed quantitative comparison of the different reconstruction results has been extended to a larger portion of the image, namely the square inscribed in the reconstruction circle (Figure 4(a)). For this region, grey value histograms have been computed. The overview plot (Figure 4(b)) clearly shows the sharp peaks (black) of the original phantom. Smaller non visible peaks are also present for values of 0.1 and 0.4 . Corresponding peaks for all reconstructions are wider, and therefore smaller. Magnified plots (Figure 4(c)-(d)) around two of the major peaks confirm that: (a) gridrec without zero padding performs rather badly (distorted non-symmetric peaks) - (b) gridrec with zero padding and FBP perform significantly better (symmetric peaks), although - (c) the peaks for FBP are slightly narrower and taller. This latter observation could arise from higher uncertainties during mapping in the Fourier space for high frequencies, where sampling is sparser. As mentioned above, for comparison purposes the results obtained with the FBP algorithm have be artificially shifted by 0.0042 .

(a)

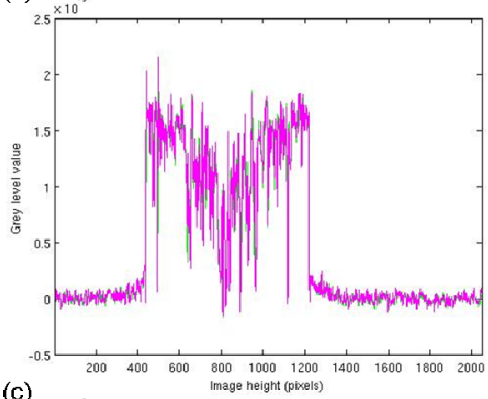

(c)

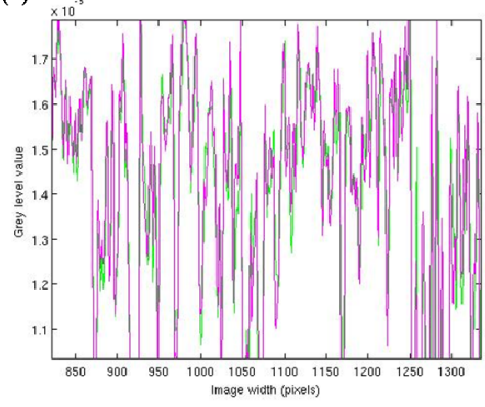

(b)

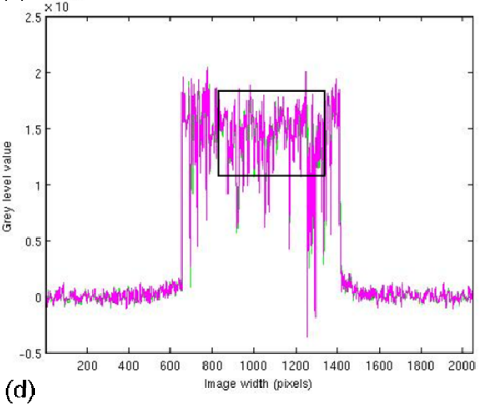

(d)

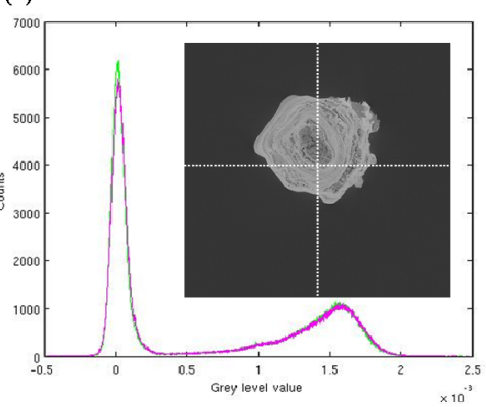

Figure 5: Accuracy assessment of the gridrec algorithm using real data (reconstructed slice shown in the inset in (d)) - (a-b) Profiles along the lines in the inset in (d) for reconstructions with FBP (green) and gridrec ( $\mathrm{Z}=1.5$, pink), (c) Magnification of the area enclosed in the black box in (b), (d) Grey level histogram. Sample: Ca Apatite kidney stone, pixel size: 1.85 micron. Sample courtesy: A. Pasch, Inselspital Bern, Switzerland. Image acquired at the TOMCAT beamline ${ }^{1}$, at the SLS-PSI, Villigen, Switzerland. 
Reconstructions of real data (Figure 5) lead to the same conclusions as for the synthetic case. Gridrec with zero padding provides high quality reconstructions barely distinguishable from FBP results, as highlighted by the magnified representation in Figure 5(c). The slight deviation noticeable here is a small offset between the two curves. Since for real data, as opposed to the synthetic example, the golden standard is not known, in this case we did not correct the FBP results for the expected a priori unknown dc shift. The high accuracy of the gridrec reconstruction is also confirmed by the histogram analysis (Figure 5(d)), where discrepancies with respect to the FBP results are minimal.

\subsection{Algorithm performance}

All reconstructions described above have been performed on a machine equipped with 2 dual core Xeon processors clocked at $3 \mathrm{GHz}$ (using though only 1 single core). Performances of the different algorithms for the reconstruction of a 2048x1501 pixel sinogram are listed in Table 1. Gridrec without zero padding, with reconstruction times of less than $1 \mathrm{~s}$, is very fast, though lacking in accuracy as shown above. Despite the observed artifacts, this very fast approach could still be useful for quick on-the-fly reconstruction of large datasets during data acquisition aimed at a first assessment of the results in particular with respect to the scan parameters. Gridrec with moderate zero padding $(\mathrm{Z}=0.5)$ provides high quality results and comparable accuracy as FBP in less than $2 \mathrm{~s}$ and is therefore more than 10 times faster than the standard FBP routine. Even gridrec with $\mathrm{Z}=1.5$, i.e. exactly the same size reconstruction as for FBP, provides results in less than $1 / 4$ of the time required by the more conventional approach.

Table 1: Algorithm performance

\begin{tabular}{lc} 
Reconstruction algorithm & Time (s) \\
\hline Gridrec - no padding & 0.77 \\
Gridrec - Z $=0.5$ & 1.92 \\
Gridrec $-\mathrm{Z}=1.5$ & 4.54 \\
FBP & 20.89 \\
\hline
\end{tabular}

\section{RING ARTIFACTS}

Rings are common artifacts in tomographic datasets. Sharp rings arise from dead pixels in CCD chips and damaged scintillator screens; instabilities of the synchrotron beam are responsible for wide and faint rings. Despite using adequate scanning protocols, high quality scintillator screens and CCD cameras, it is difficult to completely avoid such artifacts and therefore achieve highest quality reconstructions. Software routines are often required to remove the remaining distortions and thus allow full qualitative and quantitative exploitation of the acquired information.

The reconstruction algorithm implemented at TOMCAT features such a plugin, based on a new, fast and stable method, which makes use of a combined wavelet-FFT decomposition and operates in the sinogram domain, where the artifacts appear as vertical lines. Thanks to the strict condensation of the artifacts into few coefficients of the decomposed image, highly selective filtering of the unwanted features is feasible with only marginal perturbations of the true image information.

The example in Figure 6 illustrates the different steps involved in the combined wavelet-FFT decomposition approach. The original image (Figure 6(a)) is impaired by two vertical stripes. After wavelet decomposition (Figure 6(b)), the striping noise is exclusively condensed in the vertical details coefficients (red boxes). A subsequent 2D FFT of these bands (Figure 6(c)) completely condenses the unwanted signal to the abscissa (red boxes), where it can easily be filtered out, e.g. using a Gaussian function, without perturbing the useful information. The filtered image, obtained by a back transformation, is shown in Figure 6(d).

In Figure 7 this new destriping approach has been applied to synchrotron-based tomographic data. Sharp marked ring artifacts seriously deteriorate the image quality (Figure 7(a)) impairing for instance accurate segmentation. The reconstruction of the filtered sinogram reveals features and details (circled areas in Figure 7(b)) not visible in the original. 

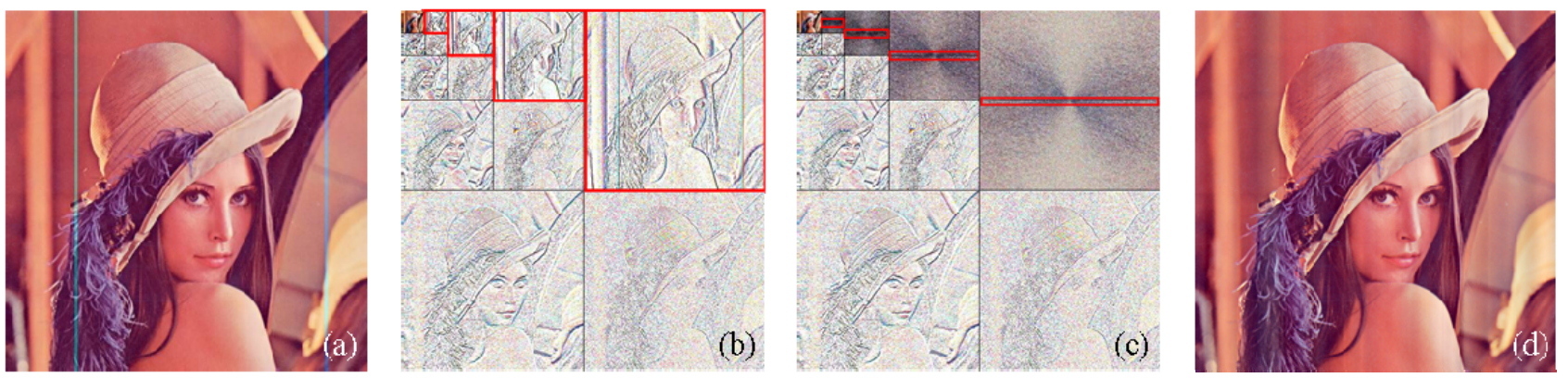

Figure 6 : Example illustrating the different steps involved in the wavelet-FFT decomposition filtering approach - (a)

Original Lena image impaired by two vertical stripes, (b) 2D multiresolution wavelet decomposition up to level 4 . The red boxes show the vertical details coefficients, where the striping noise is condensed, (c) Fourier decomposition of the vertical details coefficients. The unwanted signal is completely condensed to the abscissa (red) (d) Filtered Lenna (After Münch et al., 20095).
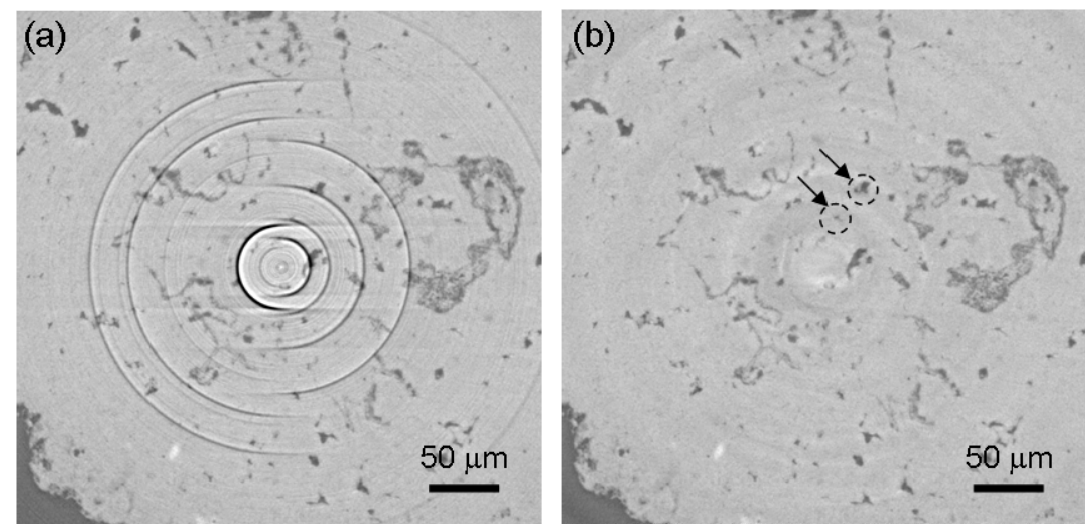

Figure 7 : Removal of sharp marked ring artifacts from a reconstructed slice with the wavelet-FFT filter - (a) Original and (b) filtered reconstruction. Sample: 530 million-year-old fossilized embryo of an animal closely related to modern marine worms (priapulids), pixel size: 0.375 microns. Sample courtesy: S. Bengtson, Swedish Museum of Natural History, Stockholm, Sweden. Image acquired at the TOMCAT beamline ${ }^{1}$, at the SLS-PSI, Villigen, Switzerland.

\section{LOCAL TOMOGRAPHY ARTIFACTS}

Often high resolution visualization and quantification of small volumes in large samples is of interest. Local tomography acquisition schemes can deliver this information, if the reconstruction approach takes into account the incompleteness of the data. In fact if local tomographic datasets are reconstructed in the standard way, large energy deposition on the reconstruction circle and a cupping artifact are usually observed (e.g. Figure 8(c), Figure 9, Figure 10(c) and Figure 11), making segmentation and quantitative analysis difficult, and strongly reducing the slice portion, which can actually be evaluated.

Artificial lateral extension of the sinogram prior to reconstruction with the aim to simulate the missing data significantly reduces typical local tomography artifacts. Here we have adopted the simplest approach and laterally padded the sinogram on both sides with the values in its first (for the left side) and last (for the right side) column. Different padding length (half $(\mathrm{Z}=0.5)$ and one and a half $(\mathrm{Z}=1.5)$ times the field of view on both sides) have been applied to synthetic and real data. In both cases, to simulate a sinogram for local tomography from a complete dataset, prior to padding the original one has been laterally cropped to $1 / 4$ of its initial size. The quality improvement achieved with the padding approach is clearly visible both in the reconstructed slices and in the line profiles: the energy deposition on the reconstruction circle is removed and the cupping artifact is strongly reduced. 
In the first example (Figure 8), the region of interest has been chosen exactly in the middle of the object. The energy deposition on the reconstruction circle and the cupping artifacts are already removed with moderate $(\mathrm{Z}=0.5)$ padding. The chosen size of the sinogram has however an influence on the absolute grey level values (Figure 9). The best results have been obtained with $Z=1.5$, which corresponds to restoring the sinogram array to its original size. Smaller $Z$ values overestimate the linear attenuation coefficient, larger $Z$ values underestimate it. This result indicates that a priori knowledge on the sample, usually present, could help in choosing the right $Z$ value.
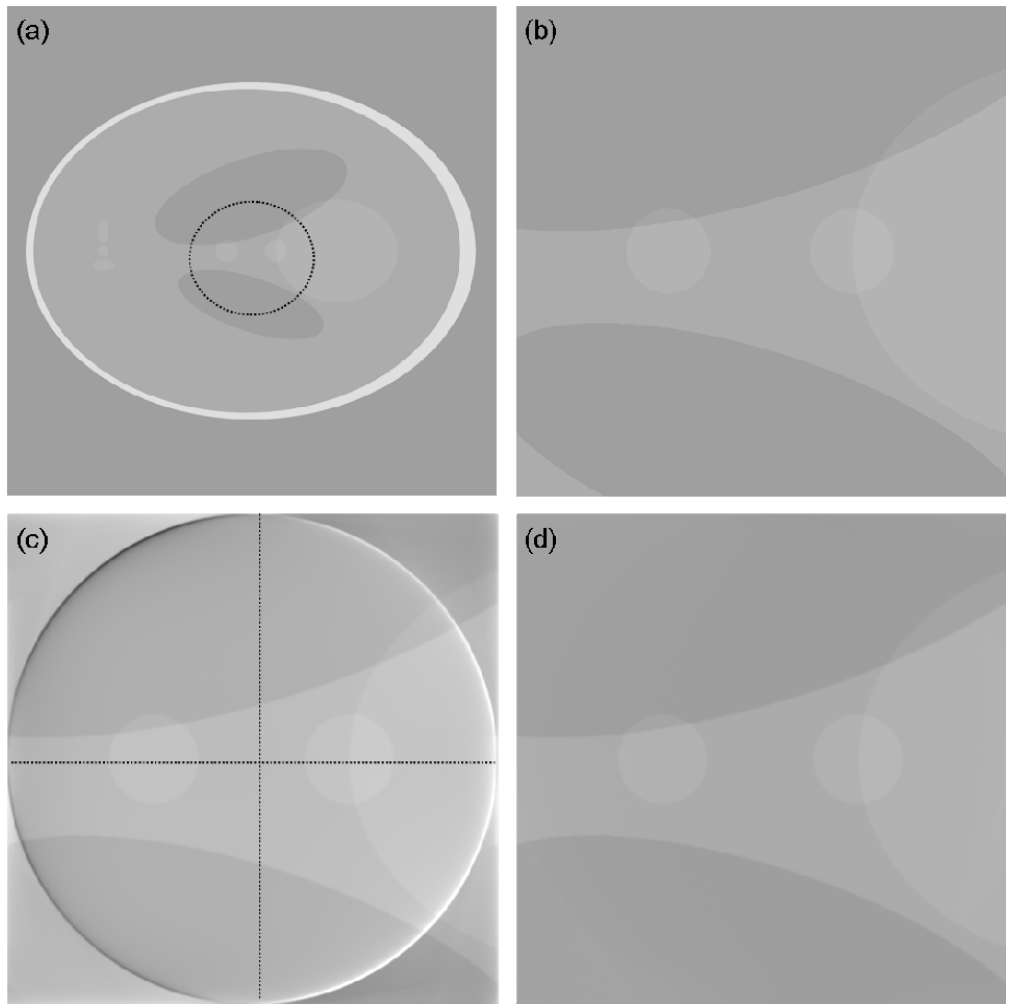

Figure 8: Reduction of local tomography artifact on a synthetic dataset - (a) Original image with the chosen region of interest indicated by the circle, (b) Magnification of the region of interest, (c) Reconstruction of the local tomography (incomplete) dataset in the standard way (the black lines show the position of the profiles in Figure 9), (d) Reconstruction of the local tomography (incomplete) dataset using sinogram padding $(\mathrm{Z}=1.5)$.

In the second example (Figure 10), the sample is occupying a relatively small portion of the field of view and the region of interest is not centered on the study object, but is located at its side. Also in this more general case, sinogram padding provides significant improvement in the image quality upon a standard reconstruction approach. Despite the size of the chosen region of interest is the same as in the previous example, for this sample the best results are obtained with $Z=0.5$ (Figure 11). This is not surprising. In fact in this second case, the sample is significantly smaller than the original field of view and its size is better approximated by doubling the sinogram array than by making it four times larger. As previously pointed out a priori sample knowledge is useful.

The presented approach is very simple. More sophisticated alternatives involving a windowed lateral extension of the sinogram with the padding values tapering to zero did not bring a significant improvement. The best results required a window with a steep flank leading to the approach proposed here. To reduce the remaining artifacts, far more complicated algorithms, involving for instance the iterative estimation of the sample transmission outside the field of view in the sinogram domain ${ }^{15}$, a wavelet-based multiresolution approach ${ }^{16}$ or other techniques ${ }^{17}$, have to be used. 
(a)

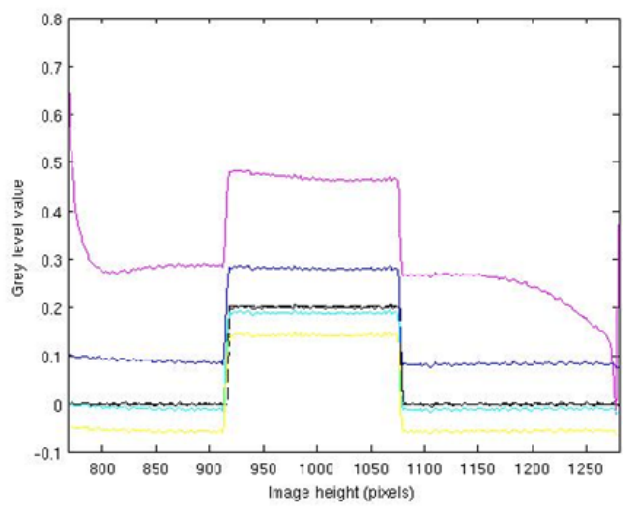

(b)

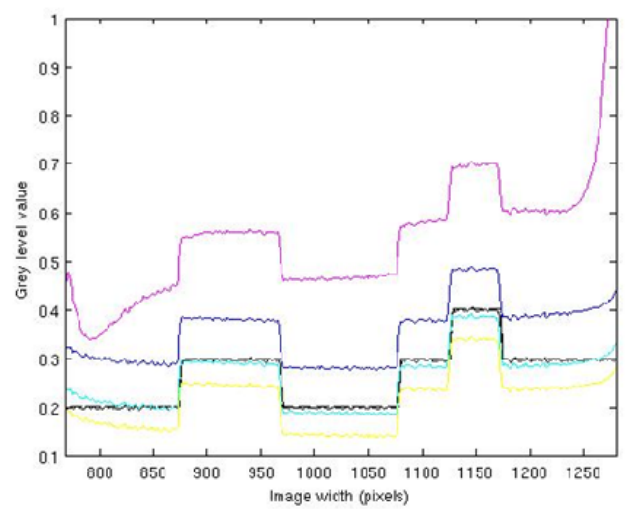

Figure 9: Reduction of local tomography artifacts on a synthetic dataset. Vertical (a) and horizontal (b) profiles along the black lines shown in Figure 8(c) through reconstructed slices obtained with different amount of sinogram padding (dash black: complete dataset, pink: standard reconstruction $(Z=0)$, blue: $Z=0.5$, cyan: $Z=1.5$, yellow: $Z=2$ ).
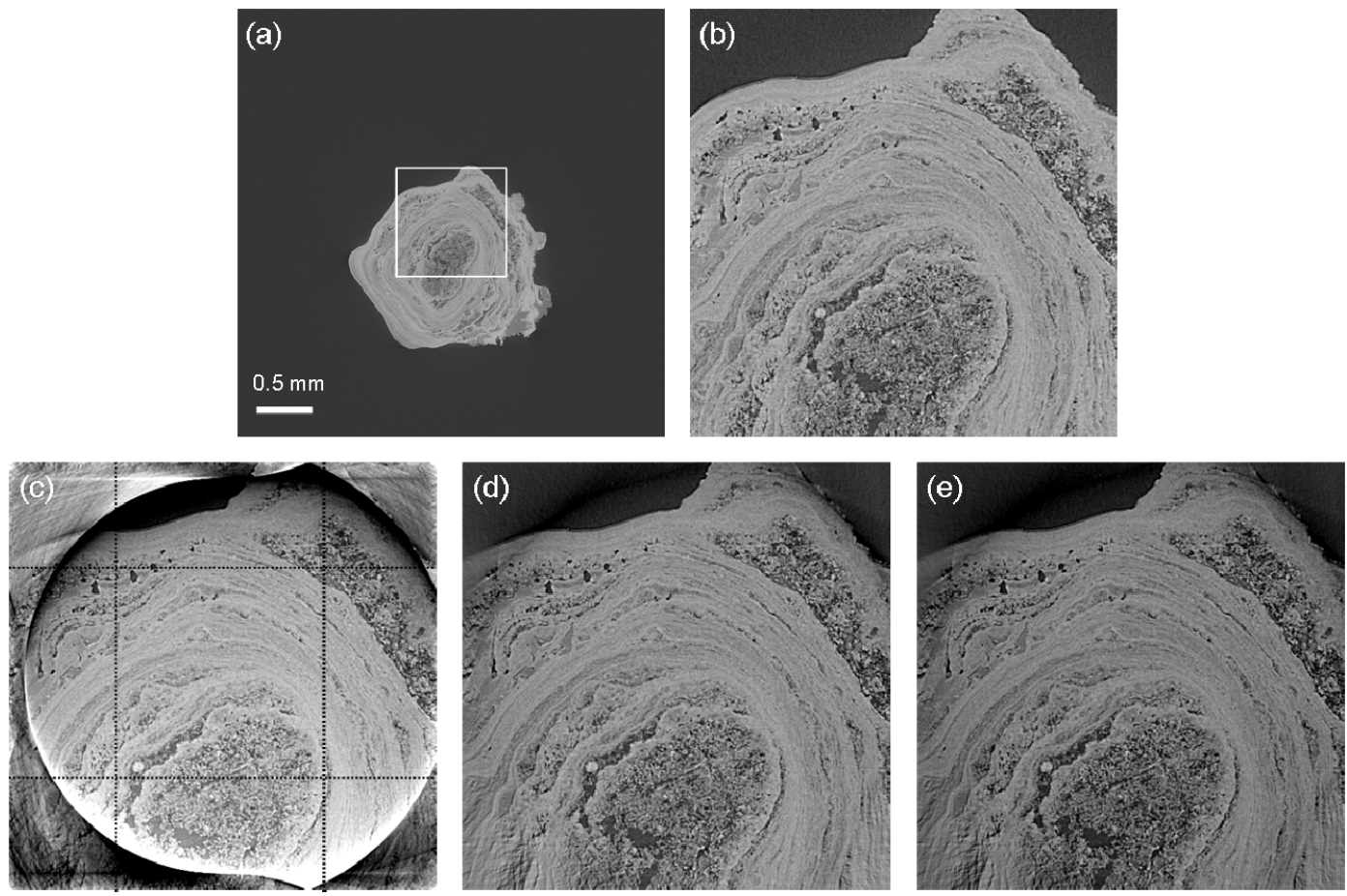

Figure 10: Reduction of local tomography artifacts on a real dataset - (a) Original image with the region of interest indicated by the white box, (b) Magnification of the region of interest. Reconstruction of the local tomography (incomplete) dataset (c) in the standard way (the black lines show the position of the profiles in Figure 11), (d) using $Z=0.5$ and (e) $\mathrm{Z}=1.5$ sinogram padding. Sample: Ca Apatite kidney stone, pixel size: 1.85 microns. Sample courtesy: A. Pasch, Inselspital Bern, Switzerland. Image acquired at the TOMCAT beamline ${ }^{1}$, at the SLS-PSI, Villigen, Switzerland. 
(a)

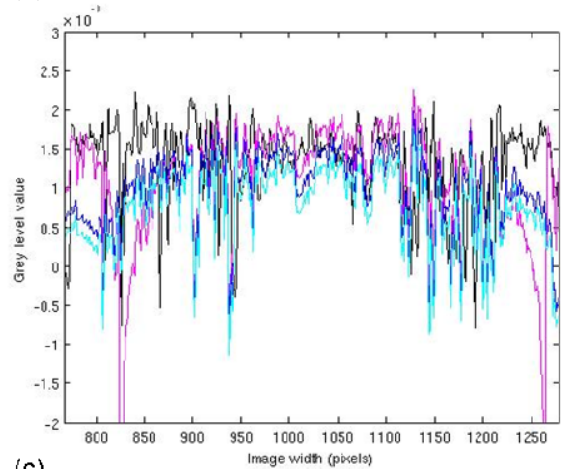

(c)

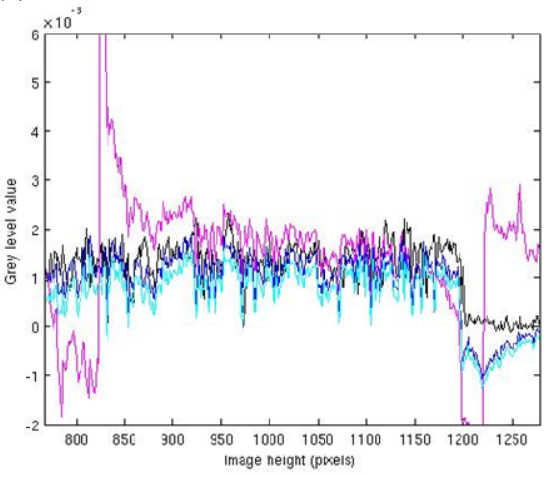

(b)

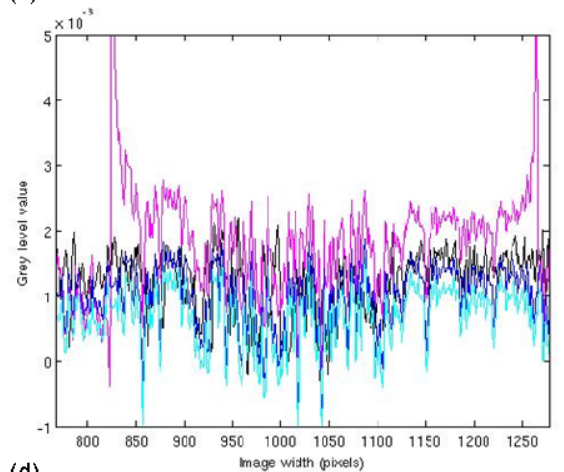

(d)

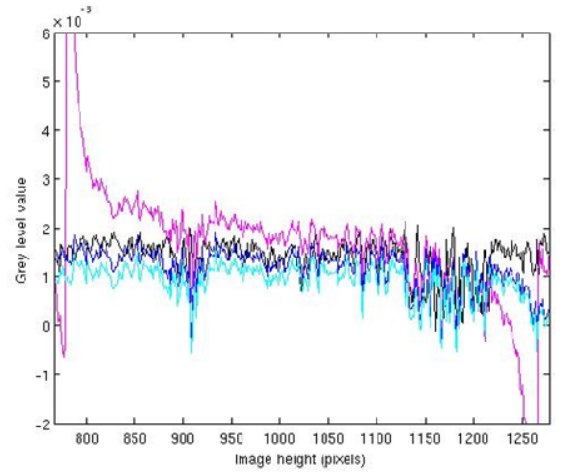

Figure 11: Reduction of local tomography artifacts on a real dataset. Horizontal (top (a) and bottom (b)) and vertical (left(c) and right(d)) profiles along the black lines shown in Figure 10(c) through reconstructed slices obtained with different amount of sinogram padding (black: complete dataset, pink: standard reconstruction $(\mathrm{Z}=0)$, blue: $Z=0.5$, cyan: $Z=1.5$ ).

\section{CONCLUSIONS}

In this paper we discuss a fast reconstruction algorithm (gridrec), originally introduces by Dowd et al. (1999) ${ }^{4}$, as an alternative to the standard Filtered Back-Projection routine. It is based on the Fourier Transform method and the delicate polar-to-Cartesian mapping in the Fourier space is performed using a gridding technique. We show that this approach guarantees high quality results coupled with a 10 fold performance increase, making real-time data post-processing closer to reality. In addition, the version implemented at the TOMCAT beamline is equipped with plugins, aimed at taming reconstruction artifacts (e.g. rings and local tomography) providing high quality results in significantly shorter time than standard FBP routines.

\section{REFERENCES}

[1] M. Stampanoni, A. Grošo, A. Isenegger et al., "Trends in synchrotron-based tomographic imaging: the SLS experience," Proc. SPIE 6318, 63180M (2006).

[2] K. Mader, F. Marone, C. Hintermüller et al., "High-throughput, fully-automatic, synchrotron-based tomographic microscopy," J. Synchrotron. Rad., submitted (2010).

[3] R. Mokso, F. Marone, and M. Stampanoni, "Real-time tomography at the Swiss Light Source," Proc. SRI (2010).

[4] B. A. Dowd, G. H. Campbell, R. B. Marr et al., "Developments in synchrotron x-ray computed microtomography at the National Synchrotron Light Source," Proc. SPIE 3772, 224-236 (1999).

[5] B. Münch, P. Trtik, F. Marone et al., "Stripe and ring artifact removal with combined wavelet - Fourier fltering," Optics Express, 17(10), 8567-8591 (2009). 
[6] F. Marone, C. Hintermuller, S. McDonald et al., "X-ray tomographic microscopy at TOMCAT," Proc. SPIE 7078, 707822 (2008).

[7] C. Hintermüller, F. Marone, A. Isenegger et al., "Image processing pipeline for synchrotron radiation-based tomographic microscopy,” J. Synchrotron Rad., 17, doi:10.1107/S0909049510011830 (2010).

[8] A. C. Kak, and M. Slaney, [Principles of computerized tomographic imaging] Society of Industrial and Applied Mathematics Philadelphia, 49-75 (2001).

[9] J. D. O'Sullivan, "A fast sinc function gridding algorithm for Fourier inversion in computer tomography," IEEE Trans. Med. Im., MI-4(4), 200-207 (1985).

[10] D. Slepian, and H. O. Pollack, "Prolate spheroidal wave functions, Fourier analysis and uncertainty - I," The Bell System Technical Journal, 40, 43-63 (1961).

[11] A. L. Van Buren, [Tables of angular spheroidal wave functions], Washington: Naval Research Laboratory (1975).

[12] M. Magnusson, P.-E. Danielsson, and P. Edholm, "Artefacts and remedies in direct Fourier tomographic reconstruction," Nuclear science symposium and medical imaging conference 2, 1138-1140 (1992).

[13] L. A. Shepp, and B. F. Logan, "The Fourier reconstruction of a head section," IEEE Trans. Nucl. Sci., 21, 21-43 (1974).

[14] R. H. Huesman, G. T. Gullberg, W. L. Greenberg et al., [RECLBL library users manual], Lawrence Berkeley Laboratory, University of California, 88 (1977)

[15] K. Mader, in preparation (2010).

[16] F. Rashid-Farrokhi, K. J. R. Liu, C. A. Berenstein et al., "Wavelet-based multiresolution local tomography," IEEE Transactions on Image Processing, 6(10), 1412-1430 (1997).

[17] A. S. Willsky, "Multiresolution Markov models for signal and image processing," Proceedings of the IEEE 90, 1396-1458 (2002). 